\title{
IGF-2 and FLT-1/VEGF-R1 mRNA Levels Reveal Distinctions and Similarities Between Congenital and Common Infantile Hemangioma
}

\author{
ARNAUD PICARD, ELISA BOSCOLO, ZIA A. KHAN, TATIANNA C. BARTCH, JOHN B. MULLIKEN, \\ MARIE PAULE VAZQUEZ, AND JOYCE BISCHOFF
}

\begin{abstract}
Departments of Surgery and Plastic Surgery [E.B., Z.A.K., T.C.B., J.B.M., J.B.], Children's Hospital, Harvard Medical School, Boston, Massachusetts 02115; and AP-HP [A.P., M.P.V.], Hôpital d'enfants Armand-Trousseau, Service de Chirurgie Maxillo-faciale et Plastique, Paris F-75012, Université Pierre et Marie Curie-Paris6, UFR de Médecine Pierre et Marie Curie, Paris, F-75005; Centre de Recherche des Cordeliers, UMRS 872 INSERM, équipe 5, Laboratoire de Biologie orale et Pathologie, Paris, F-75006, France
\end{abstract}

\begin{abstract}
Common infantile hemangioma appears postnatally, grows rapidly, and regresses slowly. Two types of congenital vascular tumors present fully grown at birth and behave differently from infantile hemangioma. These rare congenital tumors have been designated rapidly involuting congenital hemangioma (RICH) and noninvoluting congenital hemangioma (NICH). RICH and $\mathrm{NICH}$ are similar in appearance, location, and size, and have some overlapping histologic features with infantile hemangioma. At a molecular level, neither expresses glucose transporter-1, a diagnostic marker of infantile hemangioma. To gain further insight into the molecular differences and similarities between congenital and common hemangioma, we analyzed expression of insulin-like growth factor-2, known to be highly expressed in infantile hemangioma and VEGFreceptors, by quantitative real-time PCR, in three RICH and five NICH specimens. We show that insulin-like growth factor-2 mRNA was expressed in both RICH and NICH, at a level comparable with that detected in common hemangioma over $4 \mathrm{y}$ of age. In contrast, mRNA levels for membrane-associated fms-like tyrosine-kinase receptor, also known as VEGF receptor-1, were uniformly increased in congenital hemangiomas compared with proliferating or involuting phase common hemangioma. These results provide the first evidence of the molecular distinctions and similarities between congenital and postnatal hemangioma. (Pediatr Res 63: 263-267, 2008)
\end{abstract}

$\mathrm{T}$ The common infantile hemangioma is nascent in $1 \%$ to $2 \%$ of newborns, and evident in $10 \%$ of white infants by $1 \mathrm{y}$ of age. About one-third are noticed at birth, as a red macule, localized telangiectasia or blanched spot; however, the majority appear around $2 \mathrm{wk}$ after birth (1). All infantile hemangiomas exhibit postnatal growth followed by a slow yet spontaneous involution through early childhood. In contrast, the rare congenital hemangiomas are fully developed at birth and do not grow out of proportion to the infant's growth (2). Two types of congenital hemangioma have been described - each with unique clinical features and postnatal evolution. Rapidly

Received May 8, 2007; accepted October 12, 2007.

Correspondence: Joyce Bischoff, Ph.D., Vascular Biology Program, Children's Hospital Boston, 300 Longwood Avenue, Boston, MA 02115; e-mail: joyce.bischoff@ childrens.harvard.edu

Supported by the Doug and Diana Berthiaume Tribute Fund, P01 AR048564 (National Institutes of Health), Philippe Foundation Inc, (New York City, and Paris, France), AP-HP grant (Assistance Publique des Hôpitaux de Paris, Paris, France). involuting congenital hemangioma (RICH) (3) is a protuberant, hemispherical, violaceous tumor that often has a central depression, scar, or ulceration (Fig. 1A). RICH regresses by $10-14$ mo of age. Noninvoluting congenital hemangioma (NICH) (4) is well-circumscribed, plaque-like, or slightly bossed with coarse telangiectasia and often with a central or peripheral pallor (Fig. 1B). NICH never regresses, grows proportionally with the child and exhibits persistent fast-flow. Both RICH and NICH have an average diameter of 5-6 cm at birth and they do not display any sex prevalence, which is in contrast to the female preponderance in common infantile hemangioma. Mulliken and Enjolras (5) described a subset of $\mathrm{RICH}$ in which regression stops before involution is complete and the tumor persists with clinical and histopathological features of NICH. Based on these observations, they suggested that some RICH tumors transform into NICH (3).

The histopathologic characteristics of congenital hemangiomas differ from common infantile hemangioma, and from each other, but there are also overlapping features. In the proliferating phase (birth to $1 \mathrm{y}$ ), infantile hemangioma is characterized by closely approximated lobules lined by plump endothelial cells. From 1 to 2 y of age is considered the late proliferating/early involuting phase when proliferation of the tumor slows. The involuting phase $(2-5 \mathrm{y})$ is characterized by areas of persistent hyperplasia, but overall regression is seen as enlarged thin-walled channels lined by flattened endothelium appear. A progressive deposition of fibrofatty tissue leads to the involuted phase ( $>5 \mathrm{y}$ of age). Glucose transporter-1 (GLUT-1) has been shown to be a specific marker for endothelium in all phases of infantile hemangioma (6), but it is not detected in RICH or NICH (3). Histologic features of RICH are variable; there are large and small lobules separated by dense fibrous tissue, and in some lesions, there is a spongelike network of large capillaries. NICH is characterized by lobules with high cellular density: each lobule contains one or

Abbreviations: FLT-1, fms-like tyrosine-kinase receptor; GLUT-1, glucose transporter-1; HIF-1 $\boldsymbol{\alpha}$, hypoxia-inducible factor- $1 \alpha$; IGF-2, insulin-like growth factor-2; KDR, kinase-insert domain receptor; NRP, neuropilin; NICH, noninvoluting congenital hemangioma; RICH, rapidly involuting congenital hemangioma; VEGF-R, VEGF-receptor 


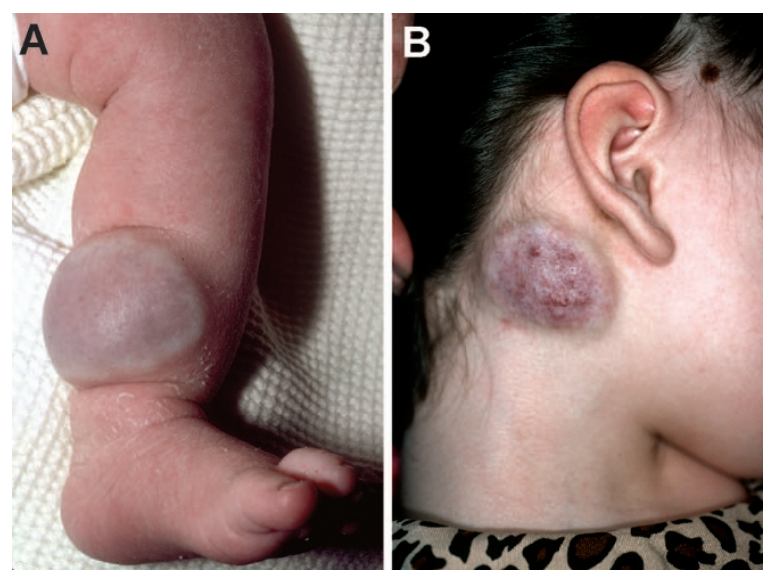

Figure 1. Clinical features of congenital hemangioma. (A) Leg: RICH: raised, purple tumor with faint halo present at birth. Fast-flow by Doppler examination. Lesion demonstrated accelerated regression. $(B)$ Neck: NICH: 3-y-old girl born with vascular tumor that has grown proportionately. Fastflow by Doppler examination.

more large, irregular intralobular vessels surrounded by multiple small vessels with indistinct lumens (3).

Except for the absence of GLUT-1 immunostaining, little is known about gene expression patterns or cellular phenotypes that might distinguish RICH and NICH from one another and each from infantile hemangioma. Insulin-like growth factor-2 (IGF-2) has been shown to be upregulated in infantile hemangioma (7) and to correlate with expression levels of VEGFreceptor-2, also known as kinase-insert domain receptor (KDR) (8). IGF-2 and GLUT-1 have been indirectly linked because IGF-2 was shown to induce hypoxia-inducible factor $1-\alpha$ (HIF- $1 \alpha$ ), and HIF- $1 \alpha$ is known to upregulate GLUT-1 $(9,10)$. To gain further insight into the relationship between congenital and common infantile hemangiomas, we investigated the mRNA and protein expression of IGF-2 and mRNA expression of the VEGF-receptors KDR (VEGF-R2), membrane associated and soluble fms-like tyrosine-kinase receptor (FLT-1) (11), neuropilin-1 (NRP-1) (12), and neuropilin-2 (NRP-2) (13). Our results show that IGF-2 is expressed at a low, but detectable levels, in both RICH and NICH. VEGFreceptors KDR, soluble FLT-1, NRP-1, and NRP-2 are expressed at variable levels in proliferating phase hemangioma $(n=3)$, involuting phase hemangioma $(n=3)$, involuted hemangioma $(n=1)$, RICH $(n=2)$, and $\mathrm{NICH}(n=5)$. However, membrane-associated FLT-1, also known as VEGF$\mathrm{R} 1$, was expressed at a consistently high level in congenital hemangioma compared with infantile hemangioma. These results provide the first demonstration of the relatively decreased expression of IGF-2 and increased expression of membrane-associated FLT-1 in congenital hemangiomas compared with common hemangioma. Hence, these findings provide molecular evidence for a close biologic relationship between the two fetal vascular tumors RICH and NICH.

\section{MATERIALS AND METHODS}

Tissue specimens. Nine resected infantile hemangiomas (ages: 5.5, 6.5, 15, $16,32,42,48,60$, and 78 mo), three resected RICH (RICH-1, 2 y; RICH-2, $1 \mathrm{mo}$; and RICH-3, 1 wk), and five resected NICH (NICH-3, 7 y; NICH-4, 6 y; NICH-5, 2.5 y; NICH-6, 28 y; and NICH-8, 3 y) were obtained under a protocol approved by the committee on Clinical Investigation, Children's Hospital Boston (Boston, MA). Newborn foreskin was obtained from the Brigham and Women's Hospital (Boston, MA) under an IRB-approved protocol. Tissue for RNA extraction from full-term placenta was kindly provided by Dr. Carmen Barnes, Children's Hospital Boston. The clinical diagnosis of common infantile hemangioma and congenital hemangioma, sub-classified as RICH or NICH, were confirmed by histologic analyses performed in the Department of Pathology, Children's Hospital Boston. Immediately after removal, specimens were embedded in optimal cutting temperature compound and snap-frozen or immersed in five volumes of RNALater ${ }^{\mathrm{TM}}$ solution (Ambion, Austin, TX) and stored at $-80^{\circ} \mathrm{C}$.

Quantitative $\boldsymbol{R} \boldsymbol{T}-\boldsymbol{P C R}$. Total RNA was isolated from proliferating, involuting, involuted, and congenital hemangioma using RNeasy Mini Kit (Qiagen, Valencia, CA) following the manufacturer's instructions. After DNAse I digestion of the RNA samples, cDNA was synthesized from total RNA with iScript cDNA Synthesis Kit (BioRad, Hercules, CA). IGF-2 and ribosomal S9 were amplified by a real-time PCR (RT-PCR) system as described (7). RT-PCR was performed for 40 cycles with SYBR Green (BioRad) using the DNA Engine Opticon 2 system (MJ Research, Inc., Waltham, MA). PCR for IGF-2 was run at $94^{\circ} \mathrm{C}$ for $30 \mathrm{~s}, 55^{\circ} \mathrm{C}$ for $30 \mathrm{~s}$, and $72^{\circ} \mathrm{C}$ for $45 \mathrm{~s}$, followed by extension at $72^{\circ} \mathrm{C}$ for $5 \mathrm{~min}$. PCR for ribosomal $\mathrm{S} 9$ was run at $94^{\circ} \mathrm{C}$ for $30 \mathrm{~s}, 55^{\circ} \mathrm{C}$ for $30 \mathrm{~s}$, and $72^{\circ} \mathrm{C}$ for $30 \mathrm{~s}$, followed by extension at $72^{\circ} \mathrm{C}$ for 5 min. A standard-curve quantification method (14) was used to determine relative levels of RNA among the tissue specimens. Cycle threshold $(\mathrm{Ct})$ values for IGF-2 and ribosomal S9, in serial dilutions of cDNA prepared from human placenta and human dermal microvascular endothelial cells, were plotted against the log of the serial dilution numbers. The resulting standard curves were used to calculate relative concentrations of IGF-2 and ribosomal S9 mRNA based on the Ct value in the different tissue specimens. RT-PCR primers for amplification of membrane FLT-1 were forward 5'-AGGGGAAGAAATCCTCCAGA-3' and reverse 5'-CGTGCTGCTTCCTGGTCC-3'; for soluble FLT-1 were forward 5'-AGGGGAAGAAATCCTCCAGA-3' and reverse 5'-CAACAAACACAGAGAAGG-3'; for NRP-1 were forward $5^{\prime}$ ACACCTGAGCTGCGGACTTT-3' and reverse 5'-GGCCTGGTCGTCATCACA-3'; and for NRP-2 forward 5'-GCGCAAGTTCAAAGTCTCCT-3' and reverse $5^{\prime}$-TCACAGCCCAGCACCTC-3'. KDR primers were as reported previously (8). The PCR amplification protocol was as follows: $94^{\circ} \mathrm{C}$ for $10 \mathrm{~s}, 53^{\circ} \mathrm{C}$ for $10 \mathrm{~s}, 72^{\circ} \mathrm{C}$ for $6 \mathrm{~s}$, and $78^{\circ} \mathrm{C}$ for $1 \mathrm{~s}$ for a total of 35 cycles.

Indirect immunofluorescence. Cryosections $(4 \mu \mathrm{m})$ of the tumors (proliferating and involuting infantile hemangioma), $\mathrm{RICH}, \mathrm{NICH}$, and pyogenic granulomas were fixed with cold acetone for $15 \mathrm{~min}$. After washing for $5 \mathrm{~min}$ in PBS, and incubating with species appropriate blocking serum, the following primary antibodies were used: mouse anti-human IGF2 MAb 1:50 (R\&D Systems, USA), mouse anti-human CD31 MAb 1:50 (DAKO, Carpinteria, CA), goat anti-human CD31 antibody 1:50 (Santa Cruz Biotechnology, Inc., CA). Normal mouse and goat-IgG were substituted for primary antibody as a negative control (same concentration as the test antibody). Primary antibodies were incubated for $1 \mathrm{~h}$ at room temperature. After washing in PBS, sections were incubated with secondary antibody for $1 \mathrm{~h}$ in the dark at room temperature. The following secondary antibodies were used: FITC-conjugated horse anti-mouse 1:200, Texas Red-conjugated horse anti-mouse 1:200, Texas Red-conjugated rabbit anti-goat 1:200 (Vector Laboratories, CA). After washing in PBS, nuclear staining was done with DAPI (Vector Laboratories) following the instructions. Sections were mounted, images examined, and captured using a Leica TCS SP2 AOBS confocal system attached to a DM IRE2 inverted microscope.

\section{RESULTS}

To compare the IGF-2 mRNA expression in infantile hemangioma with congenital hemangiomas, we measured the IGF-2 levels in a panel of RICH and NICH specimens and related these levels to that found in proliferating (age $<2 \mathrm{y}$ ), involuting (age 2-4 y), and late involuting/involuted hemangioma (age $>4$ y) (Fig. 2). The mean IGF-2 mRNA level detected in RICH was comparable with that detected in hemangiomas from children older than $4 \mathrm{y}$ of age (the late involuting phase of the hemangioma life cycle). The mean IGF-2 level in NICH specimens was lower still. Of interest is that IGF-2 mRNA was still detectable in a NICH excised at $28 \mathrm{y}$ of age. Human placental RNA was used to generate the 


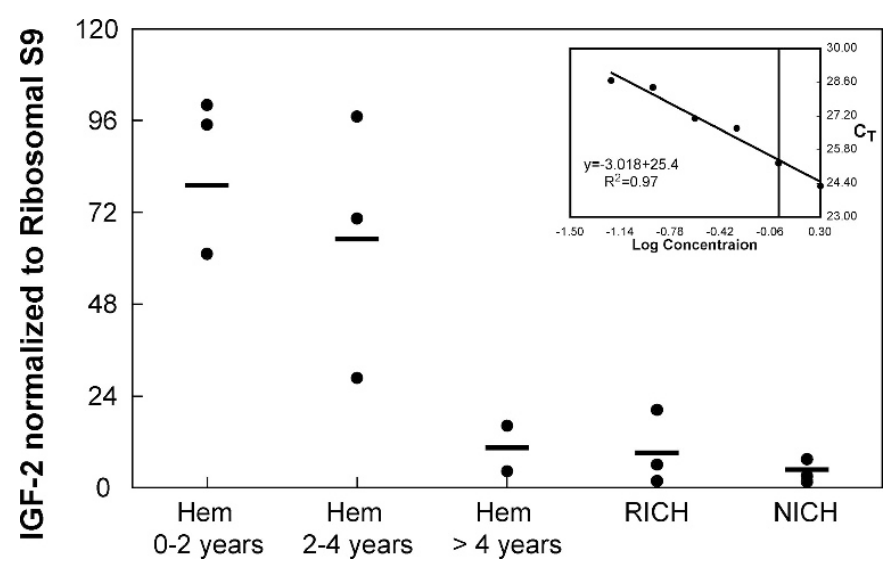

Figure 2. Quantitative real-time PCR for IGF-2. Relative IGF-2 mRNA levels are normalized to ribosomal S9 levels in common versus congenital hemangioma. Solid horizontal bars indicate average value. Mean age for $\mathrm{RICH}, 1 \mathrm{y}$; mean age for NICH, $11 \mathrm{y}$. Inset shows the IGF-2 standard curve established using RNA from human placenta.

standard curve (see inset in Fig. 2); the slope of -3.0 indicates the linearity of the assay.

To visualize IGF-2 protein expression in relation to blood vessels in congenital and infantile hemangiomas, we first immuno-stained frozen sections for CD31, an endothelial marker routinely used to detect blood vessels in tissue sections. As expected, proliferating phase hemangioma exhibited multiple regular small vessels, lined by plump CD31-positive endothelial cells (Fig. 3A). Feeding and draining vessels were indistinguishable and the CD31-positive endothelial cells were surrounded by a single layer of cells that were visualized by DAPI nuclear staining, but were CD31-negative. The NICH tissue architecture consisted of highly cellular lobules containing small vessels lined by flattened endothelial cells (Fig. 3B). Vessels with indistinct lumens surrounding larger, irregularly shaped intralobular vascular channels were also seen (Fig. 3B). The flattened endothelial cells in some of the $\mathrm{NICH}$ vessels were similar to the endothelial morphology seen in involuted hemangioma (data not shown). RICH comprised large vascular channels organized as a sponge-like network lined by moderately plump endothelium (Fig. 3C). These images are the first visualization of CD31-positive endothelial cells in NICH and RICH, and as such provide valuable insight for understanding the similarities and differences between congenital and common infantile hemangioma.

Because the data in Figure 2 show that IGF-2 mRNA was detectable in $\mathrm{RICH}$ and $\mathrm{NICH}$, we next examined the level and localization of IGF-2 protein in congenital hemangiomas by indirect immunofluorescence staining of frozen sections. Double labeling with anti-CD31 was used to determine the proximity of IGF-2 protein to blood vessels. Pyogenic granuloma was used as negative control, in accordance with our previous data (8). For comparison, IGF-2 in proliferating phase hemangioma is shown in Figure $3 D$. IGF-2 protein was detected mainly around blood vessels and in some interstitial cells; a few endothelial cells co-expressed of CD31 and IGF-2. In involuting phase hemangioma (Fig. $3 E$ ), there was a dramatic decrease in IGF-2 expression in the interstitial cells, with most of the IGF-2 localized in the single layer of cells
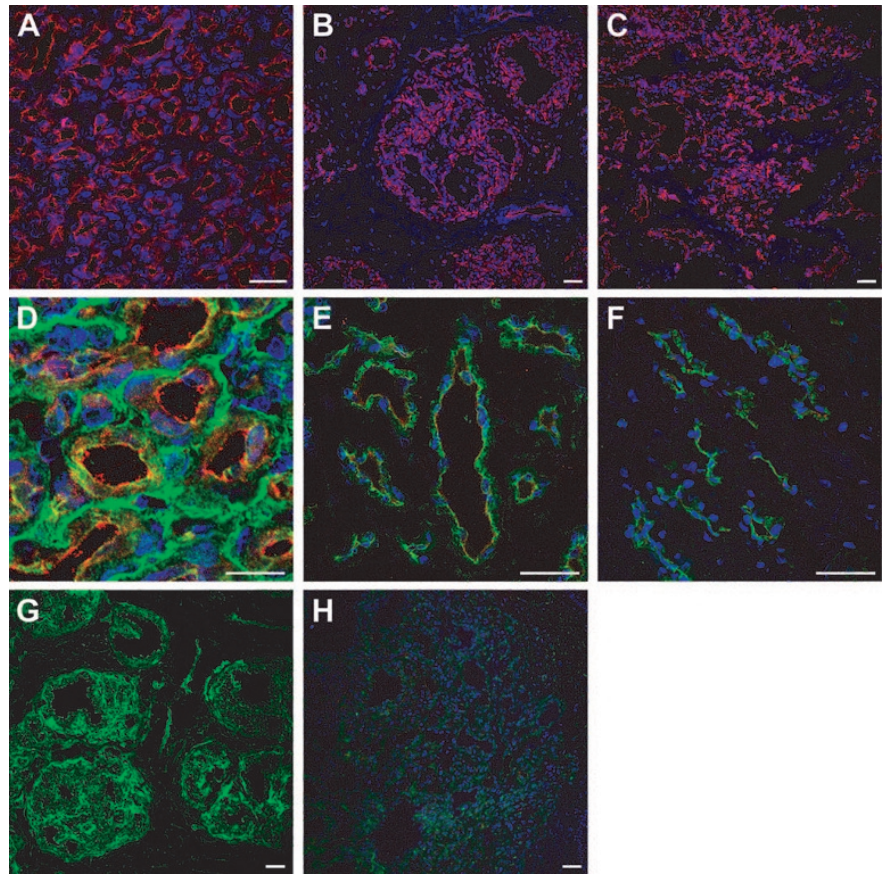

Figure 3. IGF-2 protein localized in relation to blood vessels in common and congenital hemangioma. $(A-C)$ CD31 immunostaining of frozen sections highlights blood vessels in proliferating common hemangioma $(A), \mathrm{NICH}(B)$, and RICH $(C)$. Nuclei are stained blue with DAPI. $(D-E)$ Common hemangioma sections double-labeled with anti-IGF2 (green) and anti-CD31 (red). Nuclei stained with DAPI (blue). Panel $D$, a proliferating phase hemangioma while Panel $E$, involuting phase hemangioma. $(F)$ RICH double-labeled with anti-IGF2 (green) and anti-CD31 (red). (G) NICH stained with anti-IGF2 (green). $(H)$ Pyogenic granuloma stained with anti-IGF2 (green) as negative control. Scale bars are $50 \mu \mathrm{m}$. Panel $A$ is $200 \times$ magnification; Panels $B, C$, $G$ and $H, 100 \times$ magnification; Panels $D-F, 300 \times$ magnification.

surrounding the CD31-positive endothelial cells. In RICH (Fig. $3 F$ ), IGF-2 protein expression was localized in most of the CD31-positive endothelial cells but absent from the interstitial cells. In NICH (Fig. 3G), IGF-2 was strongly expressed in the lobules around large channels. In the interlobular area, IGF-2 expression was detected in some blood vessels. This localization could not be definitely assigned to endothelial cells, as we were unable to obtain adequate co-labeling of CD31 and IGF-2 because of technical limitations. Although immuno-staining is not a quantitative tool, it is noteworthy that the fluorescent signal seen in anti-IGF-2 stained sections was comparable with that seen in proliferating phase hemangioma.

To gain further insight into the angiogenic profiles of RICH and NICH, the relative mRNA levels for the VEGF-Rs FLT-1, KDR, neuropilin-1, and neuropilin-2 were determined by quantitative RT-PCR. Total RNA was prepared from proliferating phase hemangioma $(n=3)$, involuting phase hemangioma $(n=3)$, involuted hemangioma $(n=1), \operatorname{RICH}(n=2)$ and NICH $(n=5)$ and two normal control tissues, full-term human placenta, and newborn foreskin. Expression levels were normalized to the housekeeping gene GAPDH (Fig. 4, panels $A, C-F)$. Membrane-associated FLT-1 levels are shown normalized to glyceraldehyde-phosphate dehydrogenase (Fig. $4 A$ ) and KDR (Fig. 4B). In both cases, membrane FLT-1 mRNA levels were found to be consistently higher in RICH 

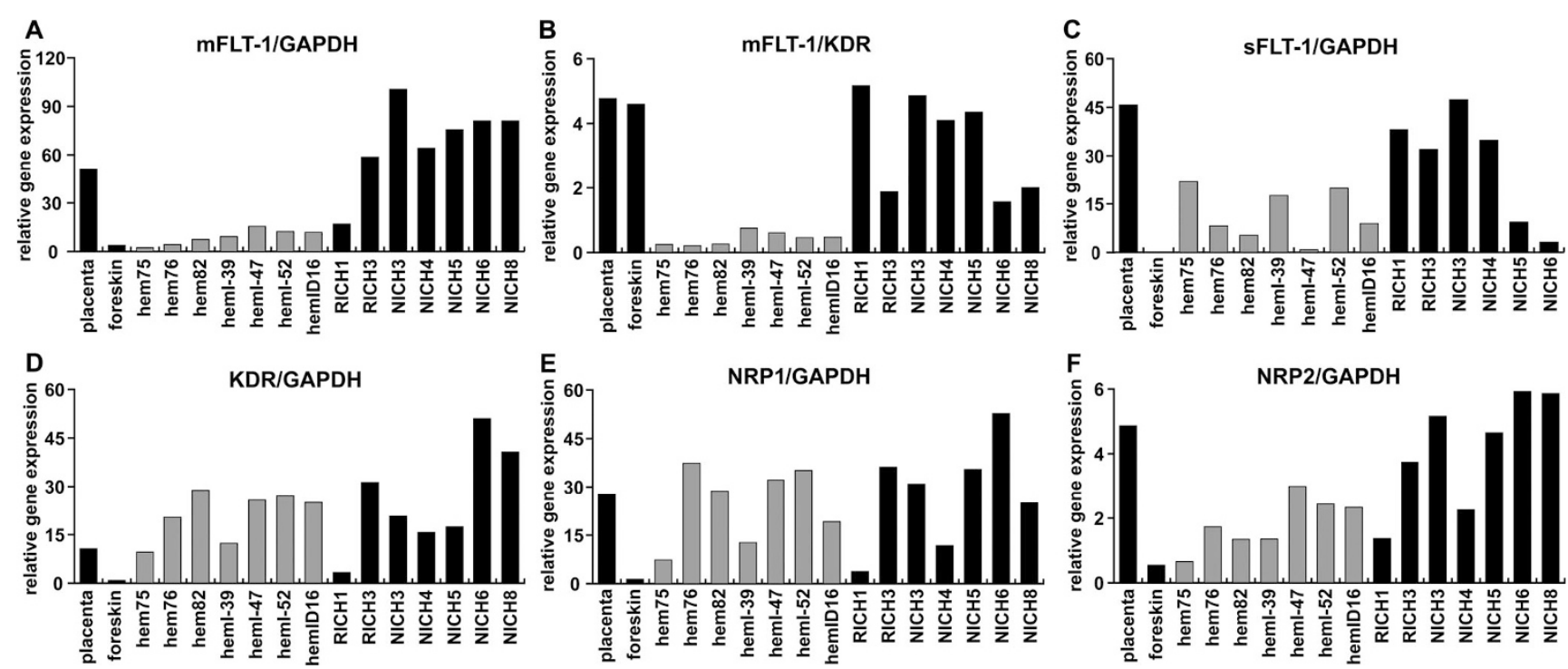

Figure 4. Real-time quantitative PCR to measure VEGF-R levels in hemangioma. Membrane FLT-1 (mFlt-1), soluble FLT-1 (sFlt-1), KDR, NRP-1, and NRP-2 mRNA transcript levels measured and normalized to glyceraldehydes phosphate dehydrogenase (GAPDH) (panels $A, C-F$ ). Level of mFLT-1 normalized to KDR levels also shown (panel $B$ ). Gray bars demarcate three proliferating phase hemangiomas (hem75, $15 \mathrm{mo}$; hem76, 6.5 mo; and hem82, 12 mo) and three involuting hemangiomas (hemI-39, $60 \mathrm{mo}$; hemI-47, $42 \mathrm{mo}$; and hemI-52, $14 \mathrm{mo}$ ), and one involuted hemangioma (hemID-16, 45 mo) from the congenital hemangiomas and from normal placenta and normal newborn foreskin (black bars).

and NICH (black bars) compared with proliferating, involuting, or involuted phase hemangioma specimens (gray bars). Membrane-associated FLT-1 levels in RICH and NICH were comparable with normal human placenta and with levels in newborn foreskin when normalized to KDR. The strikingly different pattern of expression was specific to membrane associated FLT-1, as seen in Figure 4, as levels of soluble FLT-1, KDR, NRP-1, and NRP-2 were variably expressed but with no discernible pattern among these specimens of common and congenital hemangioma specimens.

\section{DISCUSSION}

Mulliken and Enjolras (5) suggested that common infantile hemangioma and congenital hemangioma, RICH and NICH, are part of a spectrum of endothelial disruption, even though the clinical behavior is distinct in the three vascular tumors. Here, we demonstrated low but detectable levels of IGF-2 mRNA transcript in RICH and NICH, comparable with the levels found in infantile hemangioma specimens in the late involuting/involuted phase ( $>4$ y of age). Hence, based on IGF-2 expression, neither RICH nor NICH could be linked more closely to proliferating and involuting phases of common hemangioma. Furthermore, the high relative expression of FLT-1/VEGF-R1 mRNA in both RICH and NICH provides a molecular distinction from common infantile hemangioma and suggests pathways for further investigation. FLT-1 levels normalized to KDR showed that the increased FLT-1 in RICH and NICH was not due to increased endothelial content in these tumors (Fig. 4B). The relative mRNA expression levels of IGF-2 and membrane-associated FLT-1 reported here provide molecular evidence linking RICH and NICH and distinguishing RICH and NICH from common infantile hemangioma.

The histologic features of these tumors correlate with the clinical and radiological features of the vascular architecture.
Proliferating phase hemangioma is characterized by small, regular capillaries whereas congenital hemangiomas are composed of large and irregularly shaped vessels, organized into lobules in NICH and less structured in RICH. RICH can also, after several months of involution, be undistinguishable from NICH with a confluent lobular structure, although the lobules are smaller than observed in NICH $(3,4)$.

North et al. (6) showed that the endothelial cells in common infantile hemangioma express GLUT-1, yet neither RICH nor NICH are positive for GLUT-1 $(3,4)$. As GLUT-1 and IGF-2 have been indirectly linked in studies showing that IGF-2 induces HIF- $1 \alpha$, which in turn upregulates GLUT-1, we hypothesized that RICH and NICH might not express IGF-2, providing an additional means to phenotype and distinguish these variants of hemangioma. To investigate this hypothesis, we measured IGF-2 mRNA and localized IGF-2 protein in congenital hemangiomas. Our results showed that the mRNA expression of IGF-2 was detectable in RICH and NICH, but at a level comparable with that detected in older hemangioma specimens in late involuting and involuted phases. In proliferating phase hemangioma, most of the IGF-2 protein was detected in cells surrounding blood vessels. In contrast, IGF-2 was restricted to the endothelial cells in $\mathrm{RICH}$, as also has been shown for IGF-2 in involuting phase hemangioma (8). Hence, the endothelial localization of IGF-2 might indicate the onset of regression in RICH and involuting phase common hemangiomas.

IGF-2 protein in NICH specimens was localized around the large intralobular vessels and more diffusely in the small capillaries. Of note, IGF-2 mRNA was still detectable in an NICH after $28 \mathrm{y}$. In summary, we show in this study that congenital hemangiomas, fully grown at birth, express low but detectable levels of the growth factor IGF-2. However, an important limitation of this study is the small number of RICH 
and NICH tissue specimens. Although the role of IGF-2 in hemangioma-genesis remains unknown, IGF-2 is a common feature and thereby helps to characterize, at a molecular level, this spectrum of vascular tumors. Finally, the low levels of membrane-associated FLT-1 prompt the speculation that the regulation and function of this VEGF-receptor is altered in common infantile hemangioma.

Acknowledgment. We thank the following members of the Vascular Biology Program, Children's Hospital Boston: Dr. Juan Melero-Martin for advice, Jill Wylie-Sears for technical assistance, Elke Pravda for confocal microscopic imaging, Dr. Akio Shimizu for NRP-2 primer sequences, and Dr. Carmen Barnes for placental tissue RNA.

\section{REFERENCES}

1. Enjolras O, Mulliken JB 1998 Vascular tumors and vascular malformations: new issues. Adv Dermatol 13:375-423

2. Boon LM, Enjolras O, Mulliken JB 1996 Congenital hemangioma: evidence of accelerated involution. J Pediatr 128:329-335

3. Berenguer B, Mulliken JB, Enjolras O, Boon LM, Wassef M, Josset P, Burrows PE, Perez-Atayde AR, Kozakewich HP 2003 Rapidly involuting congenital hemangioma: clinical and histopathologic features. Pediatr Dev Pathol 6:495-510
4. Enjolras O, Mulliken JB, Boon LM, Wassef M, Kozakewich HP, Burrows PE 2001 Noninvoluting congenital hemangioma: a rare cutaneous vascular anomaly. Plast Reconstr Surg 107:1647-1654

5. Mulliken JB, Enjolras O 2004 Congenital hemangiomas and infantile hemangioma: missing links. J Am Acad Dermatol 50:875-882

6. North PE, Waner M, Mizeracki A, Mihm MC Jr 2000 GLUT1: a newly discovered immunohistochemical marker for juvenile hemangiomas. Hum Pathol 31:11-22

7. Ritter MR, Dorrell MI, Edmonds J, Friedlander SF, Friedlander M 2002 Insulin-like growth factor 2 and potential regulators of hemangioma growth and involution identified by large-scale expression analysis. Proc Natl Acad Sci USA 99:7455-7460

8. Yu Y, Wylie-Sears J, Boscolo E, Mulliken JB, Bischoff J 2004 Genomic imprinting of IGF2 is maintained in infantile hemangioma despite its high level of expression. Mol Med 10:117-123

9. Chen C, Pore N, Behrooz A, Ismail-Beigi F, Maity A 2001 Regulation of glut mRNA by hypoxia-inducible factor-1. Interaction between H-ras and hypoxia. J Biol Chem 276:9519-9525

10. Feldser D, Agani F, Iyer NV, Pak B, Ferreira G, Semenza GL 1999 Reciprocal positive regulation of hypoxia-inducible factor 1alpha and insulin-like growth factor 2. Cancer Res 59:3915-3918

11. Maru Y, Yamaguchi S, Shibuya M 1998 Flt-1, a receptor for vascular endothelial growth factor, has transforming and morphogenic potentials. Oncogene 16:25852595

12. Soker S, Takashima S, Miao HQ, Neufeld G, Klagsbrun M 1998 Neuropilin-1 is expressed by endothelial and tumor cells as an isoform-specific receptor for vascular endothelial growth factor. Cell 92:735-745

13. Gluzman-Poltorak Z, Cohen T, Herzog Y, Neufeld G 2000 Neuropilin-2 is a receptor for the vascular endothelial growth factor (VEGF) forms VEGF-145 and VEGF-165 [corrected]. J Biol Chem 275:18040-18045

14. Ginzinger DG 2002 Gene quantification using real-time quantitative PCR: an emerging technology hits the mainstream. Exp Hematol 30:503-512 